\title{
Human leukocyte antigen associations and c-reactive protein levels in lebanese patients with aggressive periodontitis
}

\author{
-HLA and CRP in aggressive periodontitis \\ Marita Chakhtoura, Nada M. Souccar, Nayla S. Al-Akl, Alexander M. Abdelnoor
}

Department of Microbiology \& Immunology, Faculty of Medicine, American University of Beirut, Beirut, Lebanon.

E-mail: $\underline{\text { aanoor@aub.edu.lb }}$

Received 6 May 2011; revised 16 June 2011, accepted 25 June 2011.

\begin{abstract}
Background: Periodontal disease, which affects toothsupporting structures, results from disequilibrium between the oral micro flora and host defense mechanisms. It has been classified into chronic (CP) or aggressive (AP) periodontitis according to disease onset, localization and progression. Because of their involvement in generating immune responses, Human Leukocyte Antigen (HLA) alleles are considered candidate genetic risk markers for periodontitis. Additionally, periodontitis appears to contribute to the severity of some systemic conditions such as cardiovascular disease and adverse pregnancy outcome as indicated by elevated levels of C-reactive protein (CRP). Aim: The aims of this study were to determine if there is an HLA-AP association in Lebanese patients, and to determine CRP levels in patients and compare them to those in healthy controls. Materials and methods: The study groups included 26 patients with AP and 39 healthy controls. HLA profiles were determined by DNA typing and CRP levels by ELISA. Results: HLA-A*30 (P-value $=0.010)$, HLAB*41 $\left(P_{1}=0.012\right.$ and $\left.P_{2}=0.014\right)$, HLA-DRB1*13 $\left(P_{1}\right.$ $=0.031$ and $\left.P_{2}=0.063\right)$ alleles seemed to be associated with protection against $A P$ in Lebanese patients. No linkage disequilibrium existed between alleles associated with AP. Ten of 26 AP patients (38.5\%) and 10 of $39(25.7 \%)$ controls had elevated CRP levels. Conclusion: In conclusion, protective, but no susceptible HLA alleles were detected in AP. CRP levels were not elevated in the entire AP group, and were not significantly different from controls. No linkage disequilibrium existed between alleles.
\end{abstract}

Keywords: HLA; CRP; Periodontitis; Associations

\section{INTRODUCTION}

Periodontitis, a disease affecting teeth and tooth-su- pporting structures, results from a disequilibrium between normal microbial flora and host defense mechanisms [1]. It is an inflammatory condition that is closely related to the presence of bacteria found in dental plaque, mainly Gram-negative anaerobic rods [2,3]. The disease could ultimately lead to the formation of periodontal pockets upon bone loss and apical migration of the junctional epithelium $[3,4]$. It is classified into aggressive periodontitis (AP) and chronic periodontitis (CP) $[5,6]$.

Subjects with the aggressive form have rapid attachment loss, bone loss, familial aggregation and are usually younger than 30 years of age. Those with the chronic form have at least four sites with pocket depths $>4$ $\mathrm{mm}$ and at least four sites with attachment level measurements $<4 \mathrm{~mm}$, as well as radiographic evidence of alveolar bone loss [7].

Genetic factors for periodontitis have been reviewed by Stabholz et al. [8]. The prevalence of periodontitis within families is used as a major diagnostic criterion for the aggressive form. A number of studies have indicated an association between a single nucleotide polymorphism and periodontitis. These included single nucleotide polymorphisms in the interleukin-1 gene, Fc $\gamma \mathrm{R}$ gene and the interleukin-10 gene. Genetic factors such as the Human Leukocyte Antigen (HLA) alleles, have been reported to correlate with pre-disposition to or protection against several diseases including periodontitis [9-11]. In particular, HLA-A9 and HLA-B15 seem consistently associated with aggressive periodontitis in Caucasians $[10,12,13]$, while HLA-A2, HLA-B5 and HLA-A28 are potential protective factors against periodontitis among Caucasians $[10,14]$. However, studies involving HLAperiodontitis association in the Lebanese ethnic group are lacking.

Periodontitis seems to contribute to the severity of several systemic conditions such as cardiovascular disease [15] and adverse pregnancy outcome [16,17], through elevated C-reactive protein (CRP) levels. One hypothe- 
sis suggests that this is likely achieved when inflammatory effects from periodontal disease cause oral bacterial byproducts to enter blood and trigger the production of acute phase reactants such as CRP, subsequently leading to artery inflammation or preterm birth [18]. CRP is an acute phase reactant found in plasma. Its physiological function is to enhance phagocytosis of infectious agents by macrophages. Elevated level of CRP is considered an indication of infection and inflammation. It is produced in the liver and elevated levels are thought to be due to an increase in the production of interleukin- 6 by macrophages and adiptocytes [19].

In an earlier study we reported on the detection of Porphyromonas gingivalis $(\mathrm{Pg})$ in specimens obtained from dental plaques of pregnant women with periodontitis [20]. The aims of this study were to see if HLA-AP and CRP-AP associations existed in Lebanese patients.

\section{MATERIALS AND METHODS}

\subsection{Subjects and Specimens}

A total of 65 subjects aged between 21 and 56 were recruited for this study. Twenty six patients (11 males and 15 females) with aggressive periodontitis (AP) were matched according to age, gender and smoking habits to 26 controls. Half of the controls and patients were smokers. Additional 13 controls were available and were included in the study; they were divided into 7 males and 6 females; 7 of them were smokers. Blood from all subjects was collected in citrated and plain tubes. The former was used to separate the mononuclear cells and the latter was used to obtain serum for CRP determinations.

A clinical exam that involved a full mouth periodontal probing was done on each patient and probing depth on each tooth (6 sites per tooth), bleeding on probing (BOP) [21], recessions, attachment loss (AL) [7] and Plaque index [22] were recorded. Periodontal disease was diagnosed clinically when bleeding on probing (BOP) existed in $35 \%$ or more of all tooth sites, and /or patients had at least 1 site on 4 different teeth with pocket depth $\geq 4 \mathrm{~mm}$ and clinical attachment loss $\geq 2 \mathrm{~mm}$. In addition, a radiologic examination using a panoramic or periapical radiographs to visualize disease pattern and severity, as well identify other dental pathologies was performed. Aggressive periodontitis patients were identified using the recorded measurements (including rapid attachment loss, bone loss, familial aggregation).

\subsection{Informed Consent}

Prior review and approval of this project was done by the Institutional Review Board (IRB). A written consent was obtained from each subject included in the project.

\subsection{DNA Extraction}

Peripheral Mononuclear cells (PMNC) were separated from whole blood using Ficoll-Isopaque gradient centrifugation (GE Healthcare, Pis). DNA was extracted from PMNC using the QIAamp DNA mini kit (Qiagen, Germany), according to the manufacturer's protocol.

\subsection{Determination of HLA Profiles}

HLA profiles of all patients and controls were determined by DNA typing using the polymerase chain reaction-sequence specific primer (micro-SSP) (One Lambda, Canoga Park, CA) according to the manufacturer's instructions.

\subsection{Determination of CRP Levels}

CRP levels in the sera of all participants were determined by ELISA using the high sensitivity C-reactive protein enzyme immunoassay test kit (BioCheck, Inc., Foster City, CA) according to the manufacturer's instructions. Specimens were run in duplicates and absorbance values were read at $450 \mathrm{~nm}$. CRP concentrations were calculated and expressed in mg/l. According to the manufacturers guidelines normal adult CRP values were between $0.068 \mathrm{mg} / \mathrm{l}$ and $8.2 \mathrm{mg} / \mathrm{l}$.

\subsection{Statistical Analysis}

Relative Risks (RR) and P-values were determined for each HLA allele in the AP-control groups, using Statcalc (Epi-Info, version 6.1). Linkage disequilibrium was calculated for any two associated HLA alleles using SPSS 17.0 for Windows.

Relative Risk and 95\% confidence interval (CI) were determined to see if there was a significant association between periodontitis and elevated CRP levels using the method of Hutchon, DJR [23]. An RR greater than one and a CI that does not include the number " 1 " indicated a significant association.

\section{RESULTS}

\subsection{Dental Analysis}

The dental parameters were all statistically significant difference between patient and control groups $(P \leq 0.05)$. The average values of tested indices for the patient group indicated a moderate periodontitis (Table 1).

\subsection{HLA Allele-Aggressive Periodontitis (AP) Associations}

HLA-A $* 30(P$-value $=0.010)$, HLA-B $* 41\left(P_{1}=0.012\right.$ and $\left.P_{2}=0.014\right)$ and HLA-DRB1 $* 13\left(P_{1}=0.031\right.$ and $P_{2}$ $=0.063)$ seemed to associate with protection against AP in Lebanese (Table 2). None of these alleles were in linkage disequilibrium.

\subsection{C-Reactive Protein Levels}

Ten of 26 AP patients (38.5\%) had elevated CRP levels 
(values $>8.2 \mathrm{mg} / \mathrm{l}$ ) (Table 2).

Ten of 39 individuals in the control group (25.7\%) had elevated CRP levels (values $>8.2 \mathrm{mg} / \mathrm{l}$ ) (Table 3 ). Although the number of patients with elevated CRP levels was more than that in controls, and the Relative Risk was greater than 1 (1.406), the 95\% confidence interval indicated that the association between AP and elevated CRP levels was not significant.

\section{DISCUSSION}

In determining HLA-disease associations the frequency of an allele in the diseased and normal populations are used to calculate a Relative Risk, and both populations must belong to the same ethnic group. A Relative Risk greater than 1 with $p$-value of less than 0.05 indicates an association. In normal populations some HLA allele frequencies vary among different ethnic groups and it follows that an established association in one ethnic group does not necessarily apply to another ethnic group $[24,25]$. This appears to be the case in the present study. Whereas those alleles associated with protection against AP in Caucasians were reported to be HLA-A2, HLAB5 and HLA-A28 [10,14], in Lebanese they were HLA$A * 30$, HLA-B*41 and HLA-DRB1*13. Moreover, no

Table 1. Baseline dental characteristics of all participants.

\begin{tabular}{ccc}
\hline Parameter & Study Group & Control Group \\
\hline Probing depth $(\mathrm{mm})$ & $5.3 \pm 1.9$ & $3.2 \pm 1.5$ \\
Clinical attachment loss $(\mathrm{mm})$ & $4.2 \pm 2.3$ & $1.2 \pm 0.8$ \\
& $182 / 240$ sites & $74 / 240$ sites \\
Bleeding on probing & $(75.8 \%)$ & $(30.83 \%)$ \\
\hline
\end{tabular}

Study group: periodontal disease, control group: periodontally healthy; $P \leq$ 0.05 for all parameters.

Table 2. HLA alleles associated with protection against aggressive periodontitis; AP = Aggressive periodontitis.

\begin{tabular}{cc}
\hline HLA Allele & Relative Risk and $P$-value \\
\hline HLA-A*30 & $0.27, P=0.010$ \\
& Ignored by Statcalc \\
HLA-B*41 & $P_{1}=0.012, P_{2}=0.014$ \\
& Ignored by Statcalc \\
HLA-DRB1*13 & $P_{1}=0.031, P_{2}=0.063$ \\
\hline
\end{tabular}

Table 3. Serum C-Reactive Protein (CRP) levels in Aggressive Periodontitis (AP) and Controls (C).

\begin{tabular}{ccc}
\hline \multicolumn{3}{c}{ Number of subjects } \\
\hline & with elevated CRP levels & with normal CRP levels \\
\hline Patients & 10 & 16 \\
Controls & 10 & 29 \\
\hline
\end{tabular}

Relative Risk $=1.406 ; 95 \%$ Confidence Interval $=0.78-2.53$; No significant association between elevated CRP and AP. linkage disequilibrium seemed to exist between associated alleles. The positive association of HLA-A9 and HLA-B15 with AP in Caucasians $[10,12,13]$ but not in Lebanese is also probably accounted for by the ethnic differences between the two populations. In addition, HLADRB $1 * 13$ seemed to have a more frequent occurrence in patients with AP that are Germans of Caucasian descent [26] and HLA-B*15 was also found to be associated with AP in Indian population [27].

It would be expected that all patients with AP had elevated CRP levels, but this was not the case. In the present study we did not find a significant association between elevated CRP levels and AP. Not all of the patients had elevated CRP levels and a number of periodontitis-free, apparently healthy controls had elevated CRP levels. The causes of elevated CRP levels are nonspecific and this may account for elevated levels in apparently normal controls. In as much as the patients with normal CRP levels are concerned, probably inflammation is initially confined to the oral cavity and the inflammatory mediators had not yet found their way to the liver to induce the production of CRP.

A number of reports indicated that periodontitis and elevated CRP levels were related to premature labor [15, 18]. Earlier we reported a lack of a relationship between periodontitis, elevated CRP levels and premature labor in Lebanese women [20]. It is worth noting that during the later phase of pregnancy in periodontitis-free females, CRP levels are elevated [28,29].

In conclusion, protective, but no susceptible HLA alleles were detected in AP. The association between AP and elevated CRP was statistically non-significant in Lebanese.

\section{CONFLICTING INTERESTS}

We have no conflicting interests.

\section{ACKNOWLEDGEMENTS}

This study was supported by funds from the Medical Practice Plan (MPP), Faculty of Medicine, American University of Beirut.

\section{REFERENCES}

[1] Saini, R., Marawar, P.P., Shete, S. and Saini, S. (2009) Periodontitis, a true infection. Journal of Global Infectious, 1, 149-150. doi:10.4103/0974-777X.56251

[2] Haffajee, A.D. and Socransky, S.S. (1994) Microbial etiological agents of destructive periodontal diseases. Periodontol 2000, 5, 78-111. doi:10.1111/j.1600-0757.1994.tb00020.x

[3] Li, X., Kolltveit, K.M., Tronstad, L. and Olsen, I. (2000) Systemic diseases caused by oral infection. Clinical Microbiology Reviews, 13, 547-558. doi:10.1128/CMR.13.4.547-558.2000 
[4] Cochran, D.L. (2008) Inflammation and bone loss in periodontal disease. Journal of Periodontology, 79, 1569-1576. doi:10.1902/jop.2008.080233

[5] Loesche, W.J., Grossman and N.S. (2001) Periodontal disease as a specific, albeit chronic, infection: Diagnosis and treatment. Clinical Microbiology Reviews, 14, 727-752. doi:10.1128/CMR.14.4.727-752.2001

[6] Rescala, B., Rosalem, W., Teles, R.P., Fischer, R.G., Haffajee, A., Socransky, S., Gustafsson, A. and Figueredo, C. (2010) Immunological and microbiological profiles of chronic and aggressive periodontitis subjects. Journal of Periodontology, 81, 1308-1316. doi:10.1902/jop.2010.090643

[7] Armitage, G.C. (1999) Development of a Classification System for Periodontal Diseases and Conditions. Ann Periodontol, 4, 1-6. doi:10.1902/annals.1999.4.1.1

[8] Stabholz, A., Soskolne, W.A. and Shapira, L. (2010) Genetic and environmental risk factors for chronic periodontitis and aggressive periodontitis. Periodontology 2000, 53, 138-153. doi:10.1111/j.1600-0757.2010.00340.x

[9] Reinholdt, J., Bay, I. and Svejgaard, A. (1977) Association between HLA-antigens and periodontal disease. Journal of Dental Research, 56, 1261-1263. doi:10.1177/00220345770560102901

[10] Stein, JM., Machulla, HK., Smeets, R., Lampert, F., Reichert, S. (2008) Human leukocyte antigen polymorphism in chronic and aggressive periodontitis among Caucasians: A meta-analysis. Journal of Clinical Periodontology, 35, 183-192. doi:10.1111/j.1600-051X.2007.01189.x

[11] Stein, J., Reichert, S., Gautsch, A. and Machulla, H.K. (2003) Are there HLA combinations typical supporting for or making resistant against aggressive and/or chronic periodontitis? Journal of Periodontal Research, 38, 508-517. doi:10.1034/j.1600-0765.2003.00683.x

[12] Cullinan, M.P., Sachs, J., Wolf, E. and Seymour, G.J. (1980) The distribution of HLA-A and -B antigens in patients and their families with periodontitis. Journal of Periodontal Research, 15, 177-184. doi:10.1111/j.1600-0765.1980.tb00272.x

[13] Shapira, L., Eizenberg, S., Sela, M.N., Soskolne, A. and Brautbar, H., (1994) HLA A9 and B15 are associated with the generalized form, but not the localized form, of early-onset periodontal diseases. Journal of Periodontology, 65, 219-223.

[14] Goteiner, D. and Goldman, M.J. (1984) Human lymphocyte antigen haplotype and resistance to periodontitis. Journal of Periodontology, 55, 155-158.

[15] Buhlin, K., Gustafsson, A., Pockley, A.G., Frostegard, J., and Klinge, B. (2003) Risk factors for cardiovascular disease in patients with periodontitis. European Heart Journal, 24, 2099-2107. doi:10.1016/j.ehj.2003.09.016

[16] Offenbacher, S., Beck, J.D., Lieff, S. and Slade, G. (1998) Role of periodontitis in systemic health: Spontaneous preterm birth. Journal of Dental Education, 62, 852-858.

[17] Pitiphat, W., Joshipura, K.J., Rich-Edwards, J.W., Williams P.L., Douglass C.W. and Gillman M.W. (2006) Periodontitis and plasma C-reactive protein during pregnancy. Journal of Periodontology, 77, 821-825. doi:10.1902/jop.2006.050193

[18] Kim, J. and Amar, S. (2006) Periodontal disease and systemic conditions: A bidirectional relationship. Odontology, 94, 10-21. doi:10.1007/s10266-006-0060-6

[19] Pepys, M.B. and Hirschfield, G.M. (2003) C-Reactive Protein: A critical update. Journal of Clinical Investigation, 111, 1805-1812.

[20] Souccar, N.M., Chakhtoura, M., Ghafari, J.G. and Abdelnoor, A.M. (2010) Porphyromonas gingivalis in dental plaque and serum C-reactive protein levels in pregnancy. Journal of Infections in Developing Countries, 4, 362-366.

[21] Newbrun E. (1996) Indices to measure gingival bleeding. J Periodontol, 67, 555.

[22] Silness, J. and Loe, H. (1964) Periodontal disease in pregnancy. II. Correlation between oral hygiene and periodontal condition. Acta Odontol Scand, 22, 122-135. doi:10.3109/00016356408993968

[23] Hutchon, D.J.R. (2010) Calculator for confidence intervals of relative risk. http://www.hutchon.net/ConfidRR.htm

[24] Abdelnoor, A.M. and Hunaynah, W., (1985) HLA-A,B,C typing of a selected group of Lebanese. Lebanese Medical Journal, 35, 246-250.

[25] Abdelnoor, A.M., Abdelnoor, M., Heneine, W., Khauli, R., Kobeissy, F., Mansur, S., Malak, R. and Sharara, H. (2001) Major histocompatability complex class I and class II antigens frequencies in selected groups of Lebanese. Transplantation Proceedings, 33, 2839-2840. doi:10.1016/S0041-1345(01)02211-4

[26] Reichert S, Stein, J., Fuchs, C., John, V., Schaller, H.-G. and Machulla, H.K.G. (2007) Are there common human leukocyte antigen associations in juvenile idiopathic arthritis and periodontitis? Journal of Clinical Periodontology, 34: 492-498. doi:10.1111/j.1600-051X.2007.01087.x

[27] Roshna, T., Thomas, R., Nandakumar, K. and Banerjee, M. (2006) A case-control study on the association of human leukocyte antigen-A*9 and $-\mathrm{B}^{*} 15$ alleles with generalized aggressive periodontitis in an Indian population. Journal of Periodontology, 77, 1954-1963. doi:10.1902/jop.2006.040411

[28] Miller, E.M. (2009) Changes in serum immunity during pregnancy. American Journal of Human Biology, 21, 401-403. doi:10.1002/ajhb.20882

[29] Watts, D.H., Krohn, M.A., Wener, M.H. and Eschenbach, D.A. (1991) C-reactive protein in normal pregnancy. Obstetrics \& Gynecology, 77, 176-180. doi:10.1097/00006250-199102000-00002 